\title{
Correlation between abnormal trends in the spontaneous fields of tectonic plates and strong seismicities
}

\author{
Da-Cheng Tan $\cdot$ Jian-Cun Xin
}

Received: 18 January 2017/ Accepted: 6 April 2017/Published online: 19 June 2017

(C) The Author(s) 2017. This article is an open access publication

\begin{abstract}
Tectonic activities, electrical structures, and electromagnetic environments are major factors that affect the stability of spontaneous fields. The method of correlating regional synchronization contrasts (CRSC) can determine the reliability of multi-site data trends or shortimpending anomalies. From 2008 to 2013, there were three strong earthquake cluster periods in the North-South seismic belt that lasted for 8-12 months. By applying the CRSC method to analyze the spontaneous field $\boldsymbol{E}_{\mathrm{SP}}$ at 25 sites of the region in the past 6 years, it was discovered that for each strong earthquake cluster period, the $\boldsymbol{E}_{\mathrm{SP}}$ strength of credible anomalous trends was present at minimum $30 \%$ of the stations. In the southern section of the Tan-Lu fault zone, the $\boldsymbol{E}_{\mathrm{SP}}$ at four main geoelectric field stations showed significant anomalous trends after June 2015, which could be associated with the major earthquakes of the East China Sea waters $\left(M_{\mathrm{S}} 7.2\right)$ in November 2015 and Japan's Kyushu island $\left(M_{\mathrm{S}} 7.3\right)$ in April 2016.
\end{abstract}

Keywords Spontaneous field A Anomalous trend . Reliability $\cdot$ Strong earthquake $\cdot$ Correlation

\section{Introduction}

In seismic predictions, geoelectric fields are mainly applied in disputed and short-impending precursory information

D.-C. Tan $(\bowtie) \cdot$ J.-C. Xin

Lanzhou Institute of Seismology, China Earthquake

Administration, Lanzhou, 730000, China

e-mail: tandc@gsdzj.gov.cn

D.-C. Tan

Lanzhou National Observatory Geophysics, Lanzhou 730000, China analysis (Geller 1996; Wyss et al. 1997; Uyeda 2000). Complex electromagnetic environments, dynamically rich observation data, developing observation techniques and theoretical understanding, and basic dysfunctional shortimpending predictions of catastrophic earthquakes cause credibility problems in geoelectric field observations and short-impending predictions (Geller 1996; Huang 2006).

If the confirmation of the presence of background variation trends in the geoelectric fields of the site is possible before the occurrence of catastrophic earthquakes, then the implementation of corresponding short-impending geoelectric field information analysis will have a more credible basis. Geoelectric field observation data comprise spontaneous fields, telluric fields, and interference information, which determine the necessity of reliable data analysis. The VAN method (Varotsos and Alexopoulos 1984a, b) is a short-impending earthquake prediction method based on geoelectric field anomalies, which relies on the long and short polar distances in the same location to explore seismic electric signals (SESs) and initiate predictions thereof (Ma 2008). However, the VAN method is only used for short-impending earthquake prediction with obvious controversies (Geller 1996; Huang 2006), which is based on the criticality model that electric signals are emitted when in the future earthquake focal area the gradually increasing stress reaches a critical value so that the existing electric dipoles due to defects exhibit a cooperative orientation (Varotsos 2008). In the analysis of strong regional seismicities, it is a common method for the statistical analysis of seismic catalogs. To improve the reliability of precursory seismic anomaly statistics, Huang introduced a feasible RTL (region-time-length) statistical method (Huang 2006; Huang and Liu 2006). This approach requires a relatively stable data background. In a region with intense 
tectonic activities, frequent strong quakes, and poor data stability, such as the vicinity of the North-South seismic belt in recent years, prediction using the VAN method or surveying the precursory anomalies using the RTL method is very difficult.

In the North-South seismic zone, the time quasi-synchronization phenomena occurs in the turning trends of spontaneous fields at multiple sites near the same fault zone (Tan et al. 2014). Similar phenomena also occur near the Tan-Lu fault zone. This study introduces the correlating regional synchronization contrasts (CRSC) method for determining the reliability of spontaneous field anomaly trends. Time corresponding phenomena were found between the trends of dynamic spontaneous field anomalies and strong cluster earthquakes in the statistics of the trend variation of 25 stations on the North-South seismic belt and 70 earthquakes of $M_{\mathrm{S}} 5.0$ and above from 2008 to 2013. In the Tan-Lu fault zone, correspondence existed between the trend anomalies in 2015 and increased tectonic activities in the region of Japan.

\section{Characteristics of trend variation in the spontaneous field strength}

Although the basic principle of geoelectric field observation devices is originated from the VAN method, their layout can be diverse. In general, the observation devices based on the VAN method comprise 2-3 pairs of electrodes, respectively, in the EW and NS directions. The polar distance ranges from tens to hundreds of meters. Moreover, 2-4 pairs of 1-10 km-long pole distance can be laid with appropriate configuration of their electrodes (Varotsos et al. 1991, 1993). In 1990, the Sino-French Electromagnetic Cooperation Project established the observing devices at the Songshan (SHN) station in Tianzhu, Gansu, as shown in Fig. 1a (polar distance of several kilometers was not laid). Japan Institute of Physical Chemistry's electrode layout models installed on the new isle of the Izu islands are as shown in Fig. 1b (Huang and
Liu 2006). The device comprises 8 pairs of mutually orthogonal long (several kilometers) and short (tens of meters) polar distances. Mainland China's devices are basically laid out in double-triangle shapes (also called L-shapes) as shown in Fig. 1c. The ratio of the long and short polar distances in the same direction is about 1.5 , with the long distances mostly being 300-400 m.

Geoelectric field observation data usually includes spontaneous fields, telluric fields, and interference information. $\boldsymbol{E}_{\mathrm{SP}}$ represents spontaneous field, $\boldsymbol{E}_{\mathrm{T}}$ represents telluric field, and $\boldsymbol{E}_{\mathrm{R}}$ represents the signal interference factor. The composition of the observed value of geoelectric field can be written as

$\boldsymbol{E}=\boldsymbol{E}_{\mathrm{SP}}+\boldsymbol{E}_{\mathrm{T}}+\boldsymbol{E}_{\mathrm{R}}$

When the conditions of the electromagnetic environments and the observation systems are ideal, the observed value of the $i$-th minute is set as $\boldsymbol{E}_{i}$, and the daily mean value of the strength of $\boldsymbol{E}_{\mathrm{SP}}$ calculated using the value data of minutes in one day can be expressed as formula (2). When formula (2) is applied for calculation, the relatively stable main composition of $\boldsymbol{E}_{\mathrm{T}}$, namely the effect of tidal geoelectric field, is eliminated (Tan et al. 2012):

$\boldsymbol{E}_{\mathrm{SP}} \approx \frac{1}{1440} \cdot \sum_{i=0}^{1439} \boldsymbol{E}_{i}$.

The spontaneous field on day $j$ is set as $\boldsymbol{E}_{\mathrm{SP}(j)}$, and the $\boldsymbol{E}_{\mathrm{SP}}$ daily jump can be expressed by formula (3):

$\Delta \boldsymbol{E}_{\mathrm{SP}(j)}=\boldsymbol{E}_{\mathrm{SP}(j+1)}-\boldsymbol{E}_{\mathrm{SP}(j)}$.

In general, when the determination of the impact of electromagnetic environment and observation devices at the observation site is difficult, the reliability of results calculated via formulae (2) and (3) must be confirmed. When plotting the curves of $\boldsymbol{E}_{\mathrm{SP}}$ and $\boldsymbol{\Delta} \boldsymbol{E}_{\mathrm{SP}}$, to show their long-impending trends, substantial kick data in a short duration (less than ten days) are deleted.

The observation environments and device systems of two stations in Shandan, Gansu, and Haian, Jiangsu, are favorable. Within the range of $100 \mathrm{~km}$, no earthquake of

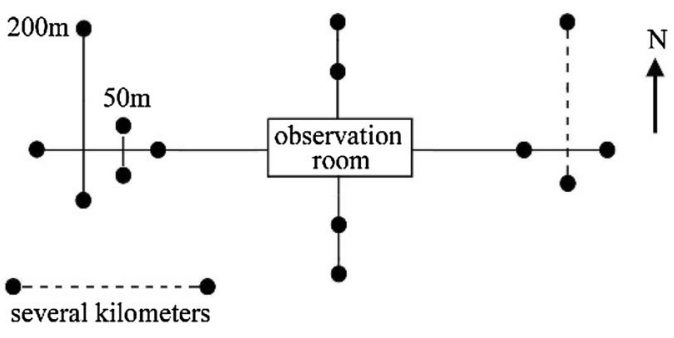

(a)

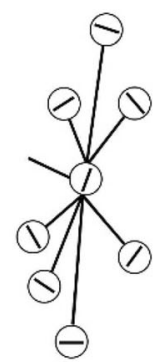

(b)

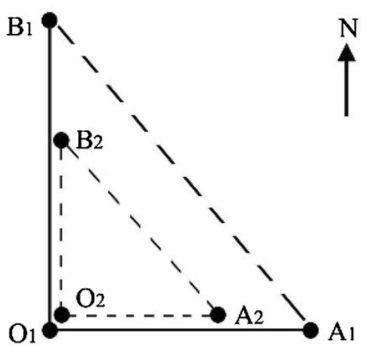

(c)

Fig. 1 Schematic of various geoelectric field orthogonal observation devices. a Orthogonal devices based on the VAN method, b orthogonal devices of Japan's new isle, and c China's orthogonal devices 
$M_{\mathrm{S}} 5.0$ or above occurred in the past 5 years. The $\boldsymbol{E}_{\mathrm{SP}}$ patterns at these two sites are stable and their annual rangeability does not exceed $100 \mathrm{mV} / \mathrm{km}$, with a slightly larger jump of $\Delta \boldsymbol{E}_{\mathrm{SP}}$ before and after the summer season, as shown in Fig. 2a, b. The locations of the two stations are shown in Fig. 2c. Figure 2a, b reflects that $\boldsymbol{E}_{\mathrm{SP}}$, and $\boldsymbol{\Delta} \boldsymbol{E}_{\mathrm{SP}}$ are relatively stable in the local areas where the earthquake activities are weak, thus showing the annual change pattern.

Figure $2 \mathrm{~d}$ indicates the two regions studied here. Figure $2 \mathrm{e}, \mathrm{f}$ is the trend curves of $\boldsymbol{E}_{\mathrm{SP}}$ and $\Delta \boldsymbol{E}_{\mathrm{SP}}$ of the Lugu lake and Luo'ci stations on the southern section of the North-South seismic belt, respectively. The locations of these two stations are shown in Fig. 2c, wherein the $\boldsymbol{E}_{\mathrm{SP}}$ and $\Delta \boldsymbol{E}_{\mathrm{SP}}$ curves show their significant range ability and violent jumps; however, the drastic changes in 2013-2014 showed better time corresponding phenomena. In recent years, strong seismicities violently occurred in the NorthSouth seismic zone. The long-term $\boldsymbol{E}_{\mathrm{SP}}$ stability of most stations in the vicinity was poor, with large magnitudes of changes and unclear cyclical annual changes, whereas phenomena like persistent fluctuation, leap, and bound existed at some $\boldsymbol{E}_{\mathrm{SP}}$ stations. However, the inflection points of their trends exhibited time quasi-synchronization with similar stability during the same period. The $\boldsymbol{E}_{\mathrm{SP}}$ patterns are irrelevant owing to site factors (Tan et al. 2014).

The southern section of the Tan-Lu fault zone mainly involves Shandong, Jiangsu, Anhui, and other provinces. The characteristics of $\boldsymbol{E}_{\mathrm{SP}}$ trend anomalies of the North-
South seismic belt also appeared in the southern section of the Tan-Lu fault zone in correspondence to the $\boldsymbol{E}_{\mathrm{SP}}$ changes of the stations in Jiashan, Anhui and Lingyang, Shandong, as shown in Fig. 3c.

\section{The reliability of spontaneous field anomalies}

3.1 Reliability analysis method for short-impending and trend anomalies of spontaneous field

At present, there are at least two methods for determining the reliability of short-impending anomalies of spontaneous fields $\boldsymbol{E}_{\mathrm{SP}}$. The first method is based on the principle of the VAN method for calculating the ratio of data variation of long-range and short-range polar distance at the same station in the same direction. If the ratio is close to one, the variation is considered to be a SES. Considering Changli station in Fig. $3 \mathrm{a}$ as an example, after zeroing treatment on the short polar distance data, the variation ranges of $\boldsymbol{E}_{\mathrm{EW}(\mathrm{L})}, \boldsymbol{E}_{\mathrm{EW}(\mathrm{S})}, \boldsymbol{E}_{\mathrm{NS}(\mathrm{L})}$, and $\boldsymbol{E}_{\mathrm{NS}(\mathrm{S})}$ during the time interval 15:54-16:13 were almost equal to each other. Such variations can be regarded as SESs (Guo et al. 2013). The second method is to conduct the correlating statistical analysis based on the seismic catalog. Figure $3 b$ lists the main steps of the RTL statistic determination method for precursory seismic anomalies (Huang 2006; Huang and Liu 2006). This method can also be applied to the reliability analysis of spontaneous field changes.

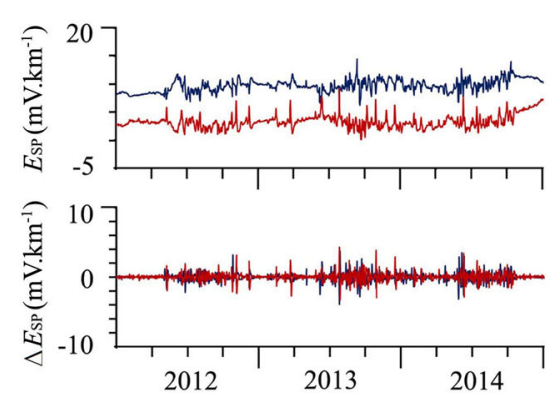

(a) Hai'an station

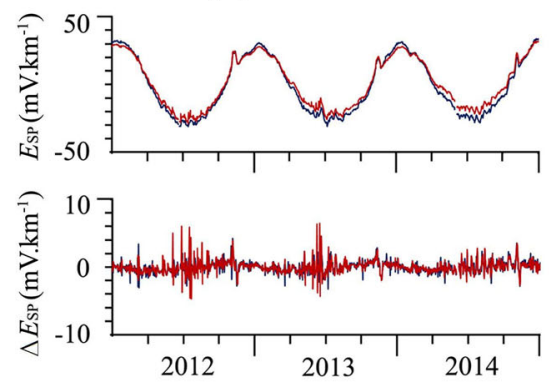

(b) Shandan station

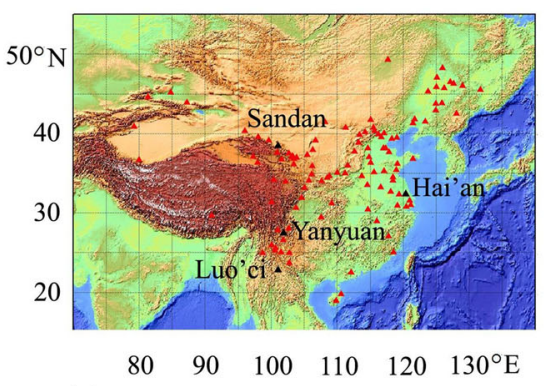

(c) Distribution of geoelectric field station

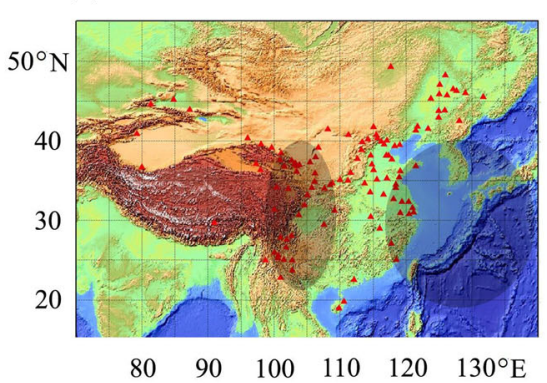

(d) Study area

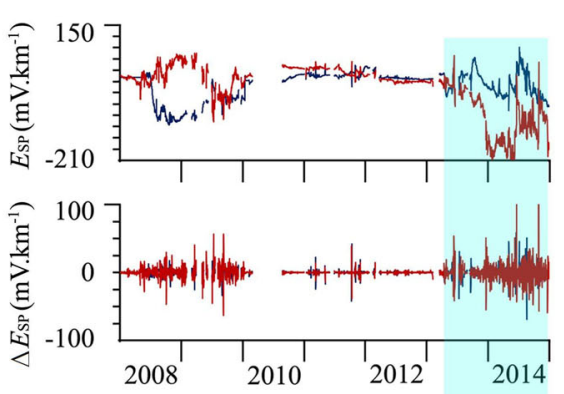

(e) Yanyuan station

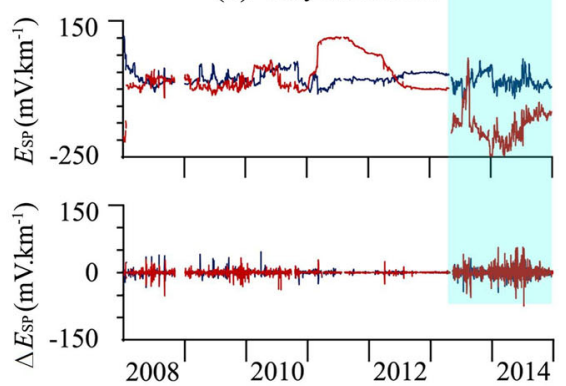

(f) Luo'ci station

Data contrast

Fig. 2 Trend variation curves of spontaneous fields $\boldsymbol{E}_{\mathrm{SP}}$ and $\boldsymbol{\Delta} \boldsymbol{E}_{\mathrm{SP}}$. a Areas with weak seismicities (2012-2014), b distribution of analysis stations, and $\mathbf{c}$ areas with strong seismicities (2008-2014) 


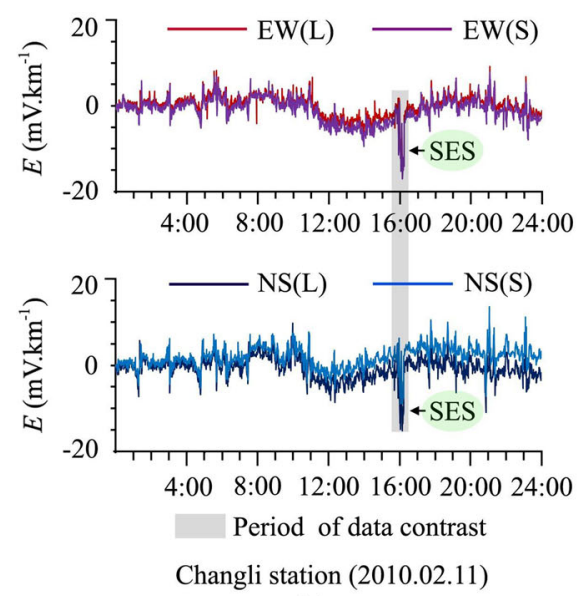

(a)

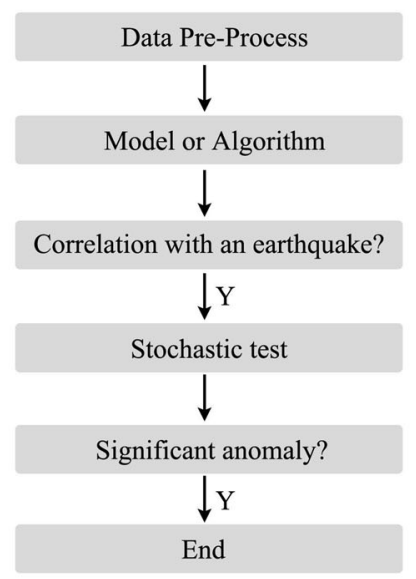

(b)

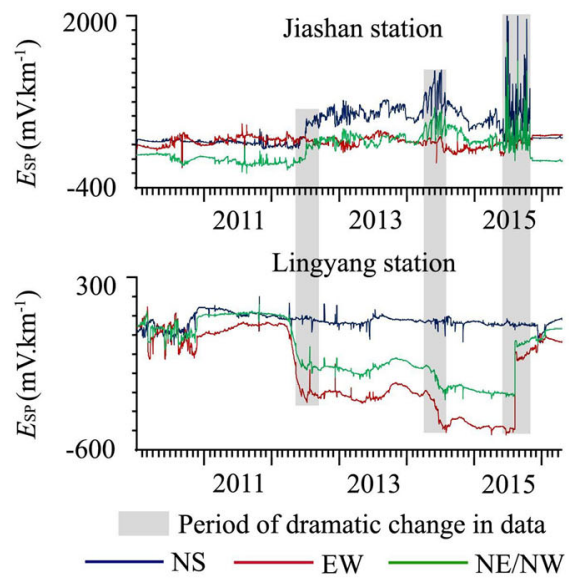

(c)

Fig. 3 Reliability analysis method for spontaneous field changes. a Application using the VAN method, b main procedures of the RTL statistical method, and $\mathbf{c}$ CRSC method

According to the VAN principle, all SESs at various sites originate from the seismic source. However, if it is assumed that the geoelectric field anomalies at each site are not from the seismic source but from the reflection of local site stress, strain, and underground fluid caused by intense local tectonic activities, then this reflection will be manifested through the geoelectric field $\boldsymbol{E}$ of the site in a large space. Moreover, there should be some differences in the anomalies of different sites in terms of $\boldsymbol{E}_{\mathrm{SP}}$ or $\boldsymbol{E}_{\mathrm{T}}$ patterns and time. For example, on the Qinghai-Tibet plateau, where tectonic activities are intense, various forms of short-impending anomalies could always be detected at more than ten remote $\boldsymbol{E}_{\mathrm{SP}}$ or $\boldsymbol{E}_{\mathrm{T}}$ stations before and after major earthquakes in recent years. The occurrence time of these anomalies exhibited quasi-synchronization (Tan et al. 2010, 2012). In recent years, studies have shown that morphological differences and time quasi-synchronization were also present in the regional trend anomalies of $\boldsymbol{E}_{\mathrm{SP}}$ and $\boldsymbol{\Delta} \boldsymbol{E}_{\mathrm{SP}}$ (Tan et al. 2014), as shown in Fig. 2e, f. In the southern section of the Tancheng-Lujiang fault zone, the $\boldsymbol{E}_{\mathrm{SP}}$ anomalies at the Jiashan and Lingyang stations are also similar, as shown in Fig. 3c.

As a result, the significant $\boldsymbol{E}_{\mathrm{SP}}$ trend and short-impending anomalies presented in this study do not have to be associated with a particular earthquake to determine their reliability. After excluding a wide range of interference factors (such as HVDC (high voltage direct current)), only the presence of the anomalies in the vicinity of the fault zone associated with the same fault zone or tectonic activities needs to be identified.

1) The $\boldsymbol{E}_{\mathrm{SP}}$ leaps, jumps, or trend anomalies at numerous sites exhibited time quasi-synchronization without entirely consistent changing patterns;

2) The intense $\Delta \boldsymbol{E}_{\mathrm{SP}}$ jumps and relatively stable changes at numerous sites exhibited time quasi-synchronization;
3) The $\boldsymbol{E}_{\mathrm{SP}}$ anomalies at certain sites and $\Delta \boldsymbol{E}_{\mathrm{SP}}$ anomalies at other sites exhibited time quasisynchronization;

4) The $\boldsymbol{E}_{\mathrm{SP}}$ and $\Delta \boldsymbol{E}_{\mathrm{SP}}$ trend anomalies at numerous sites exhibited rising and falling quasi-continuity.

When one of the above conditions is satisfied, the $\boldsymbol{E}_{\mathrm{SP}}$ anomalies at these sites are deemed to have credibility. In this study, this approach is called the correlating regional synchronization comparison method of spontaneous field changes (CRSC method). The $\boldsymbol{E}_{\mathrm{SP}}$ trend anomalies in Figs. $2 \mathrm{e}, 3 \mathrm{c}, \mathrm{f}$ can be considered to possess reliability by applying the CRSC method. It is obvious that as the number of sites that satisfy the above conditions in one region increase, the reliability of the $\boldsymbol{E}_{\mathrm{SP}}$ anomalies also increases.

It should be noted that: (1) Under the conditions of the CRSC method, the meaning of time quasi-synchronization possesses relativity. For analyzing the data of tens of days, the allowance of time quasi-synchronization can be several hours or days. For analyzing the trend variation on the year scale, the allowance of time quasi-synchronization can be tens of days. (2) The CRSC method can be applied to the reliability analysis of telluric field anomalies in principle.

\subsection{Differences and mechanisms of reliability judgment results in different methods}

Figure 2a illustrates the local regions with weak seismicities whose spontaneous fields $\boldsymbol{E}_{\mathrm{SP}}$ and $\Delta \boldsymbol{E}_{\mathrm{SP}}$ are relatively stable in general. Figures $2 \mathrm{e}, \mathrm{f}$, and $3 \mathrm{c}$ show that in the vicinity of the fault zone where tectonic activities are intense, $\boldsymbol{E}_{\mathrm{SP}}$ and $\Delta \boldsymbol{E}_{\mathrm{SP}}$ changes can be complex or without long-term stability. Since the origins of $\boldsymbol{E}_{\mathrm{SP}}$ are complex, when focusing upon analyzing the effects of different field sources, there may be differences in established physical 
models, analytical methods, and understanding of the reliability of data anomalies.

Figure $4 \mathrm{a}$ is the "point source" model diagram of the VAN method. According to the mechanism of the VAN method, SESs originate from remote seismic sources and near-field signals are an interference. The electric potential on various points around the "point electric source" is inversely proportional to the distance from the source. "Remotes sources" may cause $\Delta \boldsymbol{E}_{A 1 B 1}$ and $\Delta \boldsymbol{E}_{A 2 B 2}$ at $A_{1} B_{1}$ and $A_{2} B_{2}$ in the figure to be almost equal. According to this principle, the geoelectric field anomalies in Fig. 3a can be regarded as SESs. However, these signals did not appear subsequently on March 6 when the $M_{\mathrm{L}} 4.7$ earthquake occurred $56 \mathrm{~km}$ away from Luanxian. A possible explanation is that there were spectrum and other differences between the pre-seismic SESs and the contemporary seismic signals despite the lack of evidence. Thus, the application of long and short polar distances is mainly to explore the short-impending SESs from the seismic sources, nearfield variation of geoelectric fields, tidal waves, and distortions, which can be regarded as "interference", despite the existence of these variations (Huang and Liu 2006; Tan et al. 2010, 2011, 2012, 2014). Figure 2e shows that during 2008-2009, the EW and NE long polar distances at Yanyuan station exhibited distinctive diurnal waveforms. $\boldsymbol{E}_{\mathrm{SP}}, \boldsymbol{E}_{\mathrm{T}}$, and the adjacent Lugu lake station exhibited quasi-synchronization (Tan et al. 2010, 2011, 2012, 2014). However, the day correlation coefficients of long and short distances data in all directions were lower than 0.3. Based on the principle of the VAN method, such stations are considered to exhibit obvious near-field interference or system failure; however, the application of the CRSC law can identify that the data variations possess reliability.
In seismic case analysis, SES amplitudes mostly range from several $\mathrm{mV}$ to tens of $\mathrm{mV}$ (Ma 2008; Ma et al. 2009; Guo et al. 2013). In Fig. 3a, the amplitude of the SESs at Changli station is about $10 \mathrm{mV}$, and this lasts for about $20 \mathrm{~min}$. In the regions and time periods with intense tectonic activities, such as the period of intense $\boldsymbol{E}_{\mathrm{SP}}$ variation in Figs. 2e, f, and 3c, many large interferences can be seen based on the VAN principle, and it is difficult to find effective SESs. In fact, there are few applications of the VAN method and seismic case analyses on the NorthSouth seismic belt with large and strong earthquakes.

Intense tectonic activities will lead to very complex stress and strain inside the plate. Seismogenic areas are stress-concentrated areas. Stress and strain variation will occur on other plates or near the fault. Microfractures of rocks at the site and abnormal fluid seepage at multiple sites may occur quasi-synchronously. Therefore, at various blocks inside the plate and its adjacent plates, there is a tectonic dynamic basis for the occurrence of $\boldsymbol{E}_{\mathrm{SP}}$ and $\Delta \boldsymbol{E}_{\mathrm{SP}}$ corresponding trend changes at a number of sites (Chen et al. 2009). A study on the deep electrical structure of magnetotelluric observations (Zhao et al. 2009) indicated that the $M_{\mathrm{S}} 8.0$ Wenchuan earthquake in 2010 was not a local tectonic event but stress accumulation in the Longmenshan fault zone caused by intense tectonic activities of various locations on the Qinghai-Tibet plateau. A study on selective numerical simulation of SES sites (Huang and Lin 2010) indicated that the electrical differences of surface media affected the distribution of geoelectric fields. Based on electrokinetic effects (Ren et al. 2012, 2015) and rock fissure water (charge) seepage model of tidal geoelectric fields, significant leaps or jumps in $\boldsymbol{E}_{\mathrm{SP}}$ at the site may occur in rock mass shear fracture (Tan et al. 2014). On May

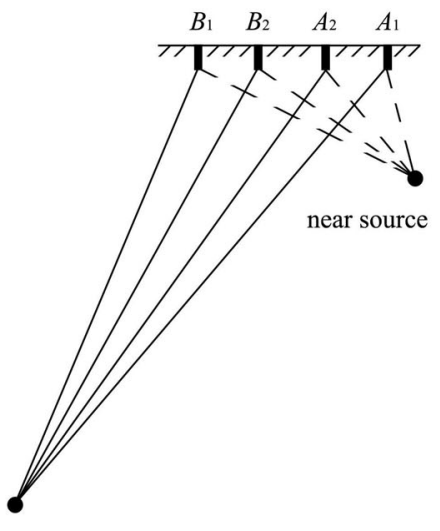

far source

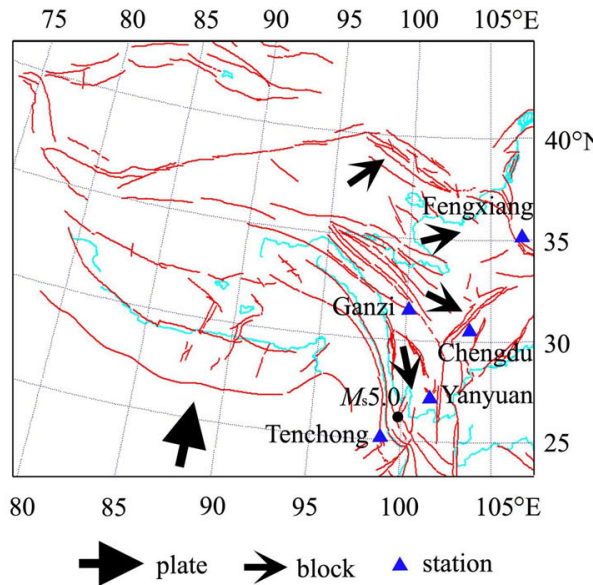

(b)

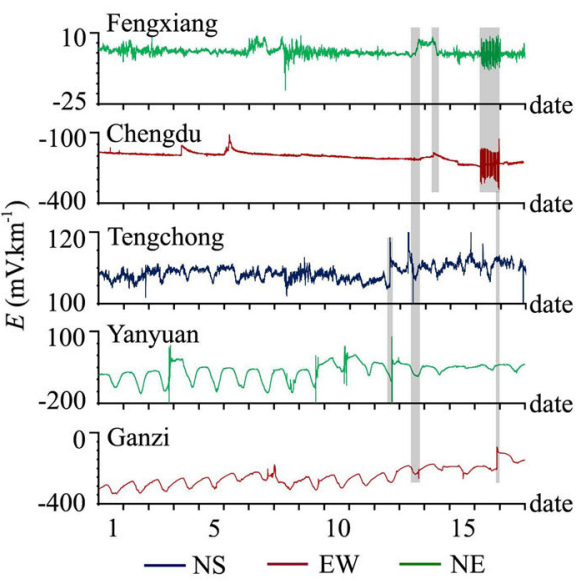

(c)

Fig. 4 Various principles for the reliability judgment methods of geoelectric field anomalies a principle of the VAN method, b tectonic plate and adjacent areas of the Qinghai-Tibet plateau, and $\mathbf{c}$ quasi-synchronous variation phenomena of geoelectric fields at different stations (2016-0501-2016-05-17) 
18, 2016, before the $M_{\mathrm{S}} 5.0$ earthquake in Yunlong County, Dali Prefecture, Yunnan, multiple stations including Fengxiang, Chengdu, Tengchong, Yanyuan, and Ganzi stations were distributed on multiple blocks, as shown in Fig. 4b, and their geoelectric field changes are shown in Fig. 4c. The step changes, jumps, and waveform distortions of the geoelectric fields at these stations possessed different levels of quasi-synchronization. Therefore, the CRSC method can obtain the support of tectonic dynamics theory, deep electrical structure research results, geoelectric field mechanism, and seismic case analysis.

It can be seen that the purpose of applying the long and short polar distance theory of the VAN method is to explore the short-impending SESs from the seismic sources. Other geoelectric field variations are deemed as interferences. The application of the CRSC method focuses on determining the authenticity of short-impending or trend anomalies of $\boldsymbol{E}_{\mathrm{SP}}\left(\boldsymbol{E}_{\mathrm{T}}\right)$ and not whether there is any direct association with earthquakes. Therefore, when analyzing

Table 1 Statistics of strong earthquakes in the region at $99^{\circ} \mathrm{E}-107^{\circ} \mathrm{E}$ and $25^{\circ} \mathrm{N}-35^{\circ} \mathrm{N}$ in recent years

\begin{tabular}{lllll}
\hline Year & $\geq M_{\mathrm{S}} 8.0$ & $\geq M_{\mathrm{S}} 7.0$ & $\geq M_{\mathrm{S}} 6.0$ & $\geq M_{\mathrm{S}} 5.0$ \\
\hline 2008 & 1 & - & 9 & 34 \\
2009 & - & - & 1 & 6 \\
2010 & - & - & 0 & 3 \\
2011 & - & - & 0 & 1 \\
2012 & - & - & 0 & 2 \\
2013 & - & 1 & 1 & 11 \\
\hline
\end{tabular}

The earthquake catalog obtained from China Earthquake Network Center the reliability of $\boldsymbol{E}_{\mathrm{SP}}$ variations with these two methods, the conclusions may be "conflicted".

\section{Correlation between the trend anomalies of spontaneous field strength and activities of strong earthquakes}

After 2008, large and strong earthquakes in mainland China were mostly concentrated near the North-South seismic belt. On April 16, 2016, a $M_{\mathrm{S}} 7.3$ earthquake occurred on Japan's Kyushu island. Therefore, this section mainly analyzes the trend variation of the spontaneous field $\boldsymbol{E}_{\mathrm{SP}}$ on the North-South seismic belt and the southern Tan$\mathrm{Lu}$ fault zone.

\subsection{North-South seismic belt}

In the vicinity of the North-South seismic belt, the longimpending stability of the spontaneous field $\boldsymbol{E}_{\mathrm{SP}}$ strength is affected by regions, sites, positions, tectonic activities, and other factors (Tan et al. 2014). Between 2008 and 2013, the statistics of the earthquakes of $M_{\mathrm{S}} 5.0$ or above occurred in the regions at $99^{\circ} \mathrm{E}-107^{\circ} \mathrm{E}$ and $25^{\circ} \mathrm{N}-35^{\circ} \mathrm{N}$ are shown in Table 1. Based on the distribution of stations in the region, the Chengdu, Hanwang, and Lugu lake stations are selected for case analysis. It should be pointed out that the observing devices and electromagnetic environments at the three stations did not experience any significant changes simultaneously during this period.

Earthquake intensity and frequency in this section are represented by colored strips. The colors of the strips in Fig. 5a represent seismic magnitudes and the widths of the (a)

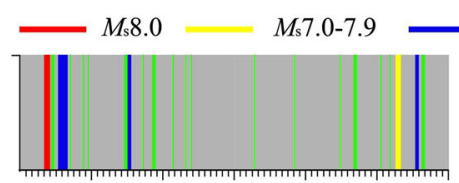

(b)

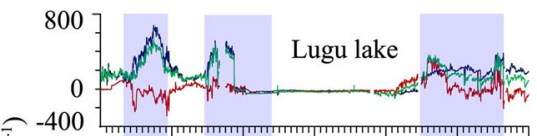

(c)

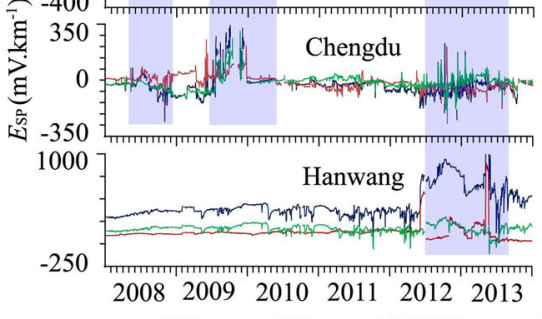

$-\mathrm{NS}-\mathrm{EW}-\mathrm{NE} / \mathrm{NW}$
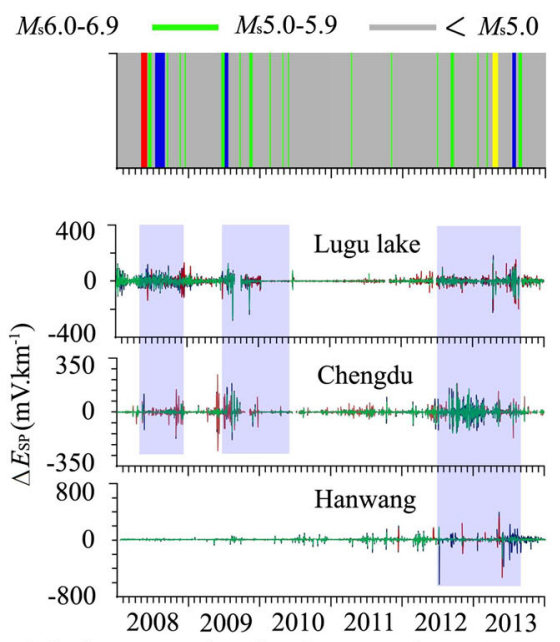

(e)

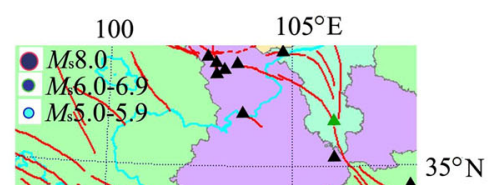

period of strong earthquake cluster $\Delta$ observatory $\Delta$ observatory of tendentious abnormal changes

Fig. 5 Correlation between natural electrical field trend anomalies and strong regional cluster earthquakes at a typical station in the North-South seismic belt (2008-2013). a Seismicity colored strips, b, c, and $\mathbf{d} \boldsymbol{E}_{\mathrm{SP}}, \boldsymbol{\Delta} \boldsymbol{E}_{\mathrm{SP}}$ curves of Lugu lake, Chengdu, and Hanwang stations, respectively, and $\mathbf{e}$ distribution of epicenters and abnormal stations in the first period (2008-05-2008-12) 
strips indicate seismic frequency. Earthquakes of $M_{\mathrm{S}} 5.0$ or above that occurred once a month are indicated by a thin line, 2-4 times by a half-month breadth line, and 5 times and above by a full-month breadth line. Multiple quakes of various magnitudes that occurred in a month are represented by the strip color determined by the highest magnitude.

Figure 5a shows the strong earthquake clusters in the regions at $99^{\circ} \mathrm{E}-107^{\circ} \mathrm{E}$ and $25^{\circ} \mathrm{N}-35^{\circ} \mathrm{N}$ that occurred in three periods from 2008 to 2013 . The first time period was from May 2008 to December 2008, the second was from June 2009 to May 2010, and the third was from September 2012 to August 2013. Figure 5a-c shows the presence of correlation between the intense changes and the relative calm of $\boldsymbol{E}_{\mathrm{SP}}$ and $\Delta \boldsymbol{E}_{\mathrm{SP}}$ at the Lugu lake and Chengdu stations. The periods of violent changes responded to the time of strong earthquake clusters. Figure 5d shows no obvious abnormality of $\boldsymbol{E}_{\mathrm{SP}}$ and $\Delta \boldsymbol{E}_{\mathrm{SP}}$ in the first and second periods at the Hanwang station. The drastic changes in the third period corresponded to the Lugu lake station and Chengdu counterparts. Therefore, the dynamic trends of $\boldsymbol{E}_{\mathrm{SP}}$ and $\Delta \boldsymbol{E}_{\mathrm{SP}}$ at the three sites are correlated with the strong seismicities of the North-South seismic belt in different degrees. Figure 5e depicts the distribution of stations with

Table 2 Statistics of $\boldsymbol{E}_{\mathrm{SP}}$ and $\Delta \boldsymbol{E}_{\mathrm{SP}}$ trend anomalies at 25 stations in the three periods of strong earthquake clusters (2008-2013)

\begin{tabular}{lclcl}
\hline Period & $\boldsymbol{E}_{\mathrm{SP}}$ & Ratio (\%) & $\boldsymbol{\Delta} \boldsymbol{E}_{\mathrm{SP}}$ & Ratio (\%) \\
\hline May 2008-Dec 2008 & 8 & 32 & 9 & 36 \\
June 2009-May 2010 & 7 & 28 & 9 & 36 \\
September 2012-August 2013 & 16 & 64 & 16 & 64 \\
\hline
\end{tabular}

abnormal trends at each strong earthquake epicenter in the first period.

Table 2 shows the statistics of 25 stations in the three periods of strong earthquake clusters shown in Fig. 5e and the numbers and ratio of stations where $\boldsymbol{E}_{\mathrm{SP}}$ and $\boldsymbol{\Delta} \boldsymbol{E}_{\mathrm{SP}}$ show abnormal trends.

It can be observed that during the three strong earthquake cluster periods on the North-South seismic belt in 2008-2013, the lowest ratio of the stations with trend anomalies in $\boldsymbol{E}_{\mathrm{SP}}$ and $\boldsymbol{\Delta} \boldsymbol{E}_{\mathrm{SP}}$ was close to $30 \%$. These trend anomalies generally exhibit quasi-synchronization similar to those in Fig. 5b-d. According to the CRSC method, the phenomenon is believed to have an acceptable reliability.

\subsection{Southern segment of the Tan-Lu fault zone}

The distribution of four main geoelectric observation stations in the southern section of the Tancheng-Lujiang fault zone is shown in Fig. 6a. From January 2012 to November 2016, no earthquake of $M_{\mathrm{S}} 5.0$ or above occurred in Jiangsu, Shandong, Anhui or other places. A $M_{\mathrm{S}} 7.2$ earthquake occurred in the East China Sea in November 2015. In April 2016, earthquakes having magnitudes of $M_{\mathrm{S}}$ $6.2, M_{\mathrm{S}} 6.0$, and $M_{\mathrm{S}} 7.3$ occurred in succession in Kyushu, Japan. Table 3 shows the statistics of earthquakes having the magnitude of $M_{\mathrm{S}} 5.0$ or above that occurred in the zones at $22^{\circ} \mathrm{N}-38^{\circ} \mathrm{N}$ and $120^{\circ} \mathrm{E}-136^{\circ} \mathrm{E}$ from January 2012 to November 2016. It can be seen that strong seismicities were clearly enhanced in 2015 and 2016.

During the period from January 2012 to November 2016, various strong earthquake epicenters occurred in the analysis areas and the locations of the geoelectric fields in Anqiu, Lingyang, Xinyi, and Jiashan stations are shown in

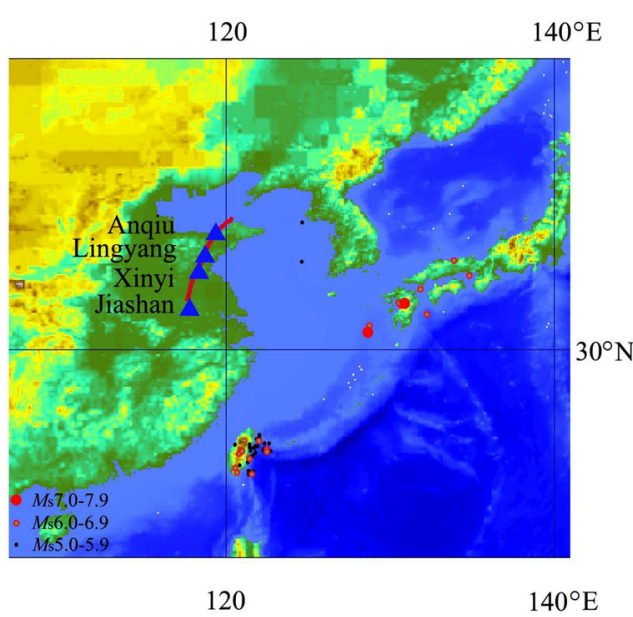

(a) $\Delta$ station of geoelectric field - Tanlu fault (b)

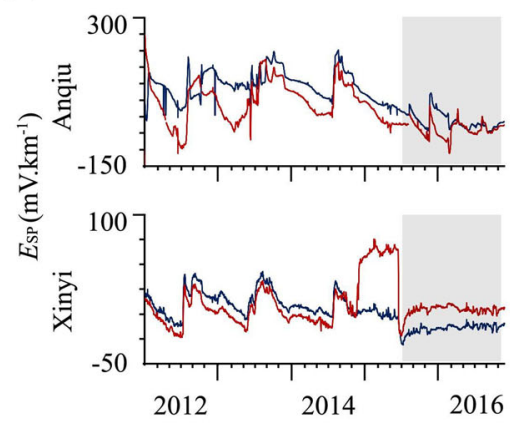

(c)
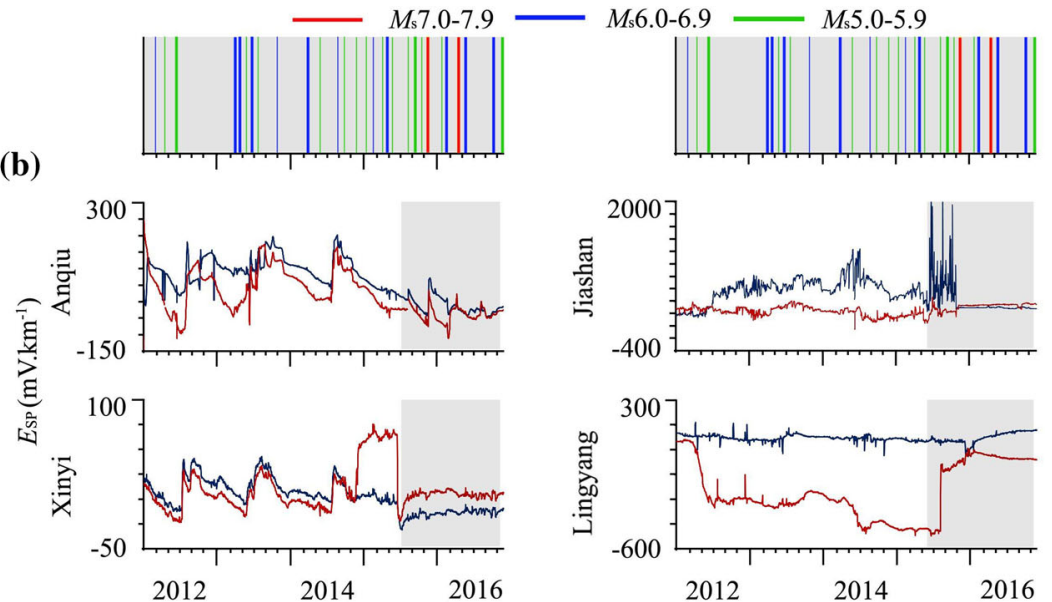

- NS - EW

Fig. 6 Spontaneous field trend anomalies of the 4th station in the southern segment of the Tan-Lu fault zone, and the contrast between the strong seismicities in the East China Sea and Kyushu island, Japan. (2012-01-01-2016-11-30). a Epicenter and station distribution, b Colored strips for strong seismicity, and c station $\boldsymbol{E}_{\mathrm{SP}}$ curve 
Table 3 Statistics of strong earthquakes in the regions at $120^{\circ} \mathrm{E}-$ $136^{\circ} \mathrm{E}, 22^{\circ} \mathrm{N}-38^{\circ} \mathrm{N}$ in recent years

\begin{tabular}{lllll}
\hline Year & $\geq M_{\mathrm{S}} 8.0$ & $\geq M_{\mathrm{S}} 7.0$ & $\geq M_{\mathrm{S}} 6.0$ & $\geq M_{\mathrm{S}} 5.0$ \\
\hline 2012 & 0 & 0 & 1 & 4 \\
2013 & 0 & 0 & 4 & 6 \\
2014 & 0 & 0 & 2 & 3 \\
2015 & 0 & 1 & 4 & 12 \\
2016 & 0 & 1 & 5 & 10 \\
\hline
\end{tabular}

Seismic catalog obtained from China Earthquake Network Center, the deadline is November 30, 2016

Fig. 6a. Figure $6 \mathrm{~b}$ shows the colored strips for strong earthquakes and their frequency in such regions, whose definitions are the same as in the previous section.

Figure $6 \mathrm{c}$ depicts the variation curve of the spontaneous field $\boldsymbol{E}_{\mathrm{SP}}$ of the four stations in the southern section of the Tan-Lu fault zone. It can be seen that after June 2015, all four $\boldsymbol{E}_{\mathrm{SP}}$ stations showed clear trend anomalies with time quasi-synchronization. According to the principle of the CRSC method, the $\boldsymbol{E}_{\mathrm{SP}}$ trend anomalies of these four stations possess reliability.

Figure 6a shows that these four stations are remote from the epicenters of strong earthquakes, whose $\boldsymbol{E}_{\mathrm{SP}}$ trend variation does not correlate with earthquakes at $M_{\mathrm{S}} 6.0$ or below. However, Fig. 6b, c shows that $\boldsymbol{E}_{\mathrm{SP}}$ trend variations have possible correlations with the major $M_{\mathrm{S}} 7.2$ earthquake in the East China Sea in November 2015, and the $M_{\mathrm{S}}$ 7.3 earthquake on Kyushu island in April 2016.

\section{Conclusions}

1) The VAN method is more suitable for the blocks or sites with relatively stable tectonic activities. Its purpose is to explore the SES for short-impending predictions since this method can narrow the time window of the impending earthquake from a few days to one week or so (Varotsos et al. 2011). The CRSC method is used for multiple sites; it is more adaptable to the regions with complex electromagnetic environments and fault distributions as well as intense tectonic activities. Moreover, it confirms the authenticity of data anomalies. The RTL statistical method depends mainly on the selection of an appropriate model or algorithm that can be broadly applied in principle. So the selection of these methods may be affected by the factors such as regional, site, tectonic activities and others in the reliability analysis of spontaneous field variations.

2) In recent years, the trend variations of spontaneous fields on the North-South seismic belt and the southern Tan-Lu fault zone have alternated between relative stability and drastic fluctuation. Overall, the spontaneous field strength of at least $30 \%$ of the stations exhibited trend anomalies during the strong earthquake cluster period on the North-South seismic belt. In the southern section of the Tan-Lu fault zone, the spontaneous field strength of four stations exhibited trend anomalies after June 2015, which might be correlated with the large earthquakes, respectively, occurred in the East China Sea in November 2015 and Kyushu island, Japan, in April 2016.

Acknowledgements We thank the reviewers and editors for their useful and careful comments. We thank the seismological bureaus of Gansu, Sichuan, Yunnan, Jiangsu, Shandong, Anhui, Hebei, Shanxi, and other provinces for providing observation data on geoelectric fields and the China Earthquake Network Center for providing the seismic catalogs. We also thank Fan Ying Ying for her help in the manuscript preparation. This study was supported by two special tasks from the Monitoring and Forecasting Department of China Earthquake Administration (16A28ZX116, 16A28ZX117).

Open Access This article is distributed under the terms of the Creative Commons Attribution 4.0 International License (http://crea tivecommons.org/licenses/by/4.0/), which permits unrestricted use, distribution, and reproduction in any medium, provided you give appropriate credit to the original author(s) and the source, provide a link to the Creative Commons license, and indicate if changes were made.

\section{References}

Chen Y, Huang TF, Liu ER (2009) Rock physics. Press of University of Science and Technology of China, Hefei

Geller RJ (1996) Debate on evalution of the VAN method. Geophys Res Lett 23(11):1291-1293

Guo JF, Ma QZ, Tong X, Zhou JQ, Xue ZF, Hu XJ (2013) Analysis on precursory abnormal characteristics of geoelectric field at Changli station. Acta Seismol Sin 35(1):50-60 (in Chinese with English abstract)

Huang QH (2006) Search for reliable precursors: a case study of the seismic quiescence of the 2000 western Tottori prefecture earthquake. J Geophys Res 111(B4):170-176

Huang QH, Lin YF (2010) Selectivity of seismic electric signal (SES) of the $2000 \mathrm{Izu}$ earthquake swarm: a 3D FEM numerical simulation model. Proc Japan Acad 86(3):257-264

Huang QH, Liu T (2006) Earthquake and tide response of geoelectric potential field at the Niijima station. Chin J Geophys 49(6):1585-1594. doi:10.1002/cjg2.986

Ma QZ (2008) Multi-dipole observation system and study on the abnormal variation of the geoelectric field observed at Capital Circle area before the Wen'an M_S5.1 earthquake. Acta Seismol Sin 06(6):618-628

Ma QZ, Zhao WG, Zhang WP (2009) Abnormal variations of geoelectric field recorded at Wenxian station preceding earthquakes and their application to the prediction of $2001 M_{\mathrm{S}} 8.1$ Kunlun earthquake. Acta Seismol Sin 31(6):660-670

Ren HX, Chen XF, Huang QH (2012) Numerical simulation of coseismic electromagnetic fields associated with seismic waves due to finite faulting in porous media. Geophys $\mathrm{J}$ Int 188(3):925-944

Ren HX, Wen J, Huang QH, Chen XF (2015) Electrokinetic effect combined with surface-charge assumption: a possible generation mechanism of coseismic EM signals. Geophys J Int 200:835-848. doi:10.1093/gji/ggu435 
Tan DC, Zhao JL, Xi JL, Du XB, Xu JM (2010) A study on feature and mechanism of the tidal geoelectrical field. Chin J Geophys 53(3):544-555. doi:10.3969/j.issn.0001-5733.2010.03.008 (in Chinese with English abstract)

Tan DC, Wang LW, Zhao JL, Xi JL, Liu DP, Yu H, Chen JY (2011) Influence factors of harmonic waves and directional waveforms for the tidal geoelectrical field. Chin J Geophys 54(4):470-484. doi:10.1002/cjg2.1630 (in Chinese with English abstract)

Tan DC, Zhao JL, Xi JL, Liu DP, An ZH (2012) The variation of waveform and analysis of composition for the geoelectrical field before moderate or strong earthquakes in Qinghai-Tibetan plateau regions. Chin J Geophys 55(2):136-149. doi:10.1002/ cjg2.1709 (in Chinese with English abstract)

Tan DC, Zhao JL, Liu XF, Fan YY, Liu J, Chen JY (2014) Features of regional variation of the spontaneous field. Chin J Geophys 57(5):1588-1598. doi:10.6038/cjg20140522

Uyeda S (2000) In defence of VAN's earthquake prediction[J]. Eos Tran. AGU 81(1):3

Varotsos $\mathrm{P}$ (2008) Point defect parameters in $\beta-\mathrm{PbF}_{2}$ revisited. Solid State Ionics 179:438-441

Varotsos P, Alexopoulos K (1984a) Physical properties of the variations of the electric field of the earth preceding earthquakes. Tectonophysics 110:73-98
Varotsos P, Alexopoulos K (1984b) Physical properties of the electric field of the earth preceding earthquakes, II. Determination of epicenter and magnitude. Tectonophysics 110:99-125

Varotsos P, Alexopoulos K, Lazaridou M (1991) Latest aspects of earthquake prediction in Greece based on seismic electric signals. Tectonophysics 188(3-4):321-347

Varotsos P, Alexopoulos K, Lazaridou M (1993) Latest aspects of earthquake prediction in Greece based on seismic electric signals II. Tectonophysics 224:1-37

Varotsos P, Sarlis NV, Skordas ES, Uyeda S, Kamogawa M (2011) Natural time analysis of critical phenomena. Proc Natl Acad Sci 108(28):11361-11364

Wyss M, Aceves RL, Park SK, Mulargia LF (1997) Cannot earthquakes be predicted? Science 278(5337):487-490. doi:10. 1126/science.278.5337.487

Zhao GZ, Chen XB, Xiao QB, Wang LF, Tang J, Zhan Y, Wang JJ, Zhang JH, Utada H, Uyeshima M (2009) Generation mechanism of Wenchuan strong earthquake of $M_{\mathrm{S}} 8.0$ inferred from EM measurements in three levels. Chin J Geophys 52(2):553-563 (in Chinese with English abstract) 